IRSTI 27.41 .41

\author{
${ }^{1}$ B.T. Zhumagulov, ${ }^{1,2}$ N.M. Temirbekov, ${ }^{3}$ Zh.R. Zhaksylykova \\ ${ }^{1}$ National Engineering Academy, Almaty, Kazakhstan \\ ${ }^{2}$ Kazakhstan engineering and technological University, \\ Almaty, Kazakhstan email: temirbekov@rambler.ru \\ ${ }^{3}$ Kazakh National Pedagogical University named after Abai, \\ Almaty, Kazakhstan email: zhaksylykova0507@mail.ru
}

\title{
Variational method for approximate solution of the Dirichlet problem
}

\begin{abstract}
Several numerical methods can be used to approximate the solution of the problem. In order to determine the most effective of them, it is necessary to carefully study each method. The most efficient approximation method is characterized by properties such as high accuracy of the solution, fewer iterations and parameters in the calculation, calculation speed, etc.

In this paper we consider the Dirichlet problem for the Poisson equation described by the initial-boundary value problem for the elliptic type of the second order. As an effective iterative method for its approximate solution, variational methods for constructing difference equations and variational methods for constructing iterative algorithms were used. The article presents the results of calculations developed using the variational method for the selected model problem. Examples of calculations for model problems are given. The results of the computational experiment demonstrate the high efficiency of the proposed iterative method.
\end{abstract}

Key words: Dirichlet problem, difference scheme, Ritz method, conjugate gradient method.

\section{Introduction}

Finding in an analytical form of the problems solution of mathematical physics is fraught with considerable mathematical difficulties. Known results apply only to the simplest cases. In other cases, are used different numerical methods of the approximate solution.

In this paper, is consider an elliptic differential equation. At the solution:

1) To construct difference equations was used a variance method, proposed in 1908 by German mathematician V. Ritz, which is called Ritz method. The solution found by this method $u_{n}(x)$, under certain conditions, tends to exact solution $u(x)$, when $n \rightarrow \infty$.

Questions of convergence of solutions obtained by the Ritz method are considered in numerous papers and monographs.

2) To construct iterative algorithms, was used the method of conjugate gradients, which stands out for its efficiency among the known iterative methods used to solve systems of linear algebraic equations.

In solving the problem, the Ritz method [1-2] was used to construct the difference equations, iterative algorithms were constructed by the method of conjugate gradients [3-4]. Comparing the results obtained by the variational method with the results obtained in the literature [4-7], it was found that the advantage of the chosen variational method is the simplicity and efficiency of memory use. Such advantages of this method will certainly be acceptable when solving large-scale problems.

\section{Variational methods for constructing difference equations.}

Consideration of the problem in general shape in the operator form

$$
L u=f, u \in \Phi(L)
$$


when $\Phi(L)$ - is an operator domain $L$

This task is equivalent to the corresponding variational problem.

$$
J(u)=\min _{v \in \Phi(L)} J(v),
$$

when $J(v)=(L v, v)-2(f, v)$.

Consideration of the Ritz method use in solving an elliptic differential equation of the form

$$
-\sum_{i, j=1}^{2} \frac{\partial}{\partial x_{i}}\left(A_{i j}(x) \frac{\partial u}{\partial x_{j}}\right)=f(x), \text { in } D
$$

with boundary condition

$$
\left.u\right|_{\partial D}=0
$$

where $D$ is a bounded domain with a piecewise linear boundary $\partial D, A_{i j}(x)=A_{j i}(x)$ bounded functions and for an arbitrary vector $\xi=\left(\xi_{1}, \xi_{2}\right)^{\prime}$ is done inequality

$$
\mu_{0} \sum_{i=1}^{2} \xi_{i}^{2} \leq \inf _{x \in D} \sum_{i, j=1}^{2} A_{i j}(x) \xi_{i} \xi_{j} \leq \sup _{x \in D} \sum_{i, j=1}^{2} A_{i j}(x) \xi_{i} \xi_{j} \leq \mu_{1} \sum_{i=1}^{n} \xi_{i}^{2}
$$

with positive constants $\mu_{0} \leq \mu_{1}$.

It can be shown that the operator of problems (3) and (4) is symmetric and positive definite, and the problem itself reduces to finding a function that minimizes in space $\stackrel{\circ}{W}$

$$
J(u)=\int_{D}\left(\sum_{i, j=1}^{2} A_{i j}(x) \frac{\partial u}{\partial x_{i}} \frac{\partial u}{\partial x_{j}}\right) d D-2 \int_{D} u f d D
$$

To find an approximate solution of problem (5), is applied the Ritz method with special-type $F_{h}$ subspaces that satisfy condition (4).

After, we construct $F_{h}$ subspaces. To simplify the presentation, it is illustrated by the example of piecewise linear approximations, when domain
$D=\left\{\left(x_{1}, x_{2}\right): 0<x_{1}, x_{2}<1\right\}$ is a square. This area is covered with a uniform square grid with a pitch $h=\frac{1}{N+1}$ and then divide each of the squares $D_{k, l}$ with diagonal. All the internal vertices of the triangles are numbered through $\left\{p_{k, l}\right\}_{k, l=1}^{N}$, the union of all triangles with a point of its vertex $p_{k, l}$ mark through $D_{k, l}^{h}$.

$D_{k, l}^{h}$ can be represented as a union of six triangles $\left\{D_{k, l, m}^{h}\right\}_{m=1}^{6}$, the order of numbering is indicated in fig.1

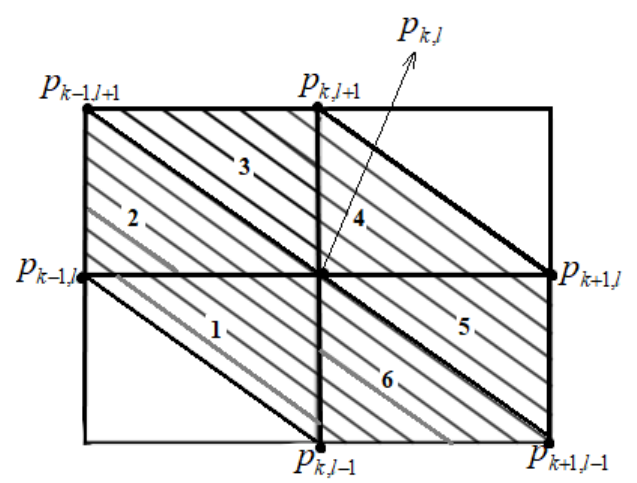

Figure 1 - $\left\{D_{k, l, m}^{h}\right\}_{m=1}^{6}$ triangle designation

To each internal node $\left(x_{k}, y_{l}\right), k, l=\overline{1, N}$ of the grids is assigned a piecewise linear basic function $\varphi_{k, l}(x, y)$. We define each of these functions $\varphi_{k, l}(x, y)$ for the entered grid.

To set $\varphi_{k, l}(x, y)$ analytical, enough for each triangle entering the carrier $\varphi_{k, l}(x, y)$, to create an equation for the plane passing through the unit in $p_{k, l}$, and in the other two of its vertices through zero. Calculating basic functions $\varphi_{k, l}(x, y)$ in each of triangles $\left\{D_{k, l, m}^{h}\right\}_{m=1}^{6}$ we build a system of basic functions 


$$
\varphi_{k, l}(x, y)=\left\{\begin{array}{l}
1-\frac{1}{h}\left(x_{k}-x\right)-\frac{1}{h}\left(y_{l}-y\right),(x, y) \in D_{k, l, 1}^{h}, \\
1-\frac{1}{h}\left(x_{k}-x\right),(x, y) \in D_{k, l, 2}^{h}, \\
1+\frac{1}{h}\left(y_{l}-y\right),(x, y) \in D_{k, l, 3}^{h}, \\
1+\frac{1}{h}\left(x_{k}-x\right)+\frac{1}{h}\left(y_{l}-y\right),(x, y) \in D_{k, l, 4}^{h}, \\
1+\frac{1}{h}\left(x_{k}-x\right),(x, y) \in D_{k, l, 5}^{h}, \\
1-\frac{1}{h}\left(y_{l}-y\right),(x, y) \in D_{k, l, 6}^{h} .
\end{array}\right.
$$

To build an approximate $u^{h}(x)$ solution of the problem (1) and (2) we apply Ritz method using basis $\left\{\varphi_{k, l}(x)\right\}_{k, l=1}^{N}$

$$
u^{h}(x)=\sum_{k, l=1}^{N} \alpha_{k, l} \varphi_{k, l}(x)
$$

As a result, comes a system of linear equations

$$
A \alpha=g
$$

where $\alpha=\left(\alpha_{1}, \alpha_{2}, \ldots, \alpha_{N^{2}}\right)^{T}$ - vector, made up from decomposition coefficients $\left\{\alpha_{N(k-1)+l}=\alpha_{k, l}\right\}_{k, l=1}^{N}$, $g=\left(g_{1}, g_{2}, \ldots, g_{N^{2}}\right)^{T}$ - vector with components

$$
g_{N(k-1)+l}=g_{k, l}=\int_{D_{k, l}} f \varphi_{k, l}(x) d D, k, l=\overline{1, N}
$$

and the elements of matrix A are calculated by the formulas

$$
\begin{gathered}
a_{k, l}^{i, j}=a_{N(k-1)+l, N(i-1)+j}= \\
=\int_{D} \sum_{s, t=1}^{2} A_{s t}(x) \frac{\partial \phi_{k, l}}{\partial x_{s}} \frac{\partial \phi_{i, j}}{\partial x_{t}} d D, \quad k, l, i, j=\overline{1, N}
\end{gathered}
$$

We consider Dirichlet problem in the area $D$ with border $\partial D$ with variable coefficients $p(x, y)$ and $q(x, y)$

$$
-\frac{\partial}{\partial x} p(x, y) \frac{\partial u}{\partial x}-\frac{\partial}{\partial y} q(x, y) \frac{\partial u}{\partial y}=f(x, y), \text { in } D(11
$$

with boundary condition

$$
\left.u\right|_{\partial D}=0
$$

The equation (10) is multiplied by the function $u(x, y)$ and integrated by $D$ in parts, given the boundary condition (12) we obtain

$$
\int_{D} p(x, y)\left(\frac{\partial u}{\partial x}\right)^{2} d D+\int_{D} q(x, y)\left(\frac{\partial u}{\partial y}\right)^{2} d D=\int_{D} f(x, y) \cdot u d D
$$

According to (5) we construct the functional

$$
J(u)=\int_{D} p(x, y)\left(\frac{\partial u}{\partial x}\right)^{2} d D+\int_{D} q(x, y)\left(\frac{\partial u}{\partial y}\right)^{2} d D-2 \int_{D} f(x, y) u d D
$$

To apply the Ritz method in (13) the function $u(x, y)$ replacing by decomposition (7) we get 


$$
\begin{gathered}
J\left(u^{h}\left(\alpha_{k, l}\right)\right)=\int_{D} p(x, y)\left(\frac{\partial}{\partial x} \sum_{k, l=1}^{N} \alpha_{k, l} \varphi_{k, l}(x)\right)^{2} d D+\int_{D} q(x, y)\left(\frac{\partial}{\partial y} \sum_{k, l=1}^{N} \alpha_{k, l} \varphi_{k, l}(x)\right)^{2} d D- \\
-2 \int_{D} f(x, y) \sum_{k, l=1}^{N} \alpha_{k, l} \varphi_{k, l}(x) d D, \quad k, l=1, \ldots, N
\end{gathered}
$$

Next, the derivatives are found and equating to zero, we get the following equations

$$
\begin{gathered}
\frac{\partial J\left(u^{h}\left(\boldsymbol{\alpha}_{k, l}\right)\right)}{\partial \boldsymbol{\alpha}_{k, l}}=\int_{D} p(x, y)\left(\sum_{k, l=1}^{N} \boldsymbol{\alpha}_{k, l} \frac{\partial \boldsymbol{\phi}_{k, l}(x)}{\partial x}\right) \frac{\partial \boldsymbol{\phi}_{k, l}(x)}{\partial x} d D+ \\
+\int_{D} q(x, y)\left(\sum_{k, l=1}^{N} \boldsymbol{\alpha}_{k, l} \frac{\partial \boldsymbol{\phi}_{k, l}(x)}{\partial y}\right) \frac{\partial \boldsymbol{\phi}_{k, l}(x)}{\partial y} d D-\int_{D} f(x, y) \boldsymbol{\phi}_{k, l}(x) d D=0, \quad k, l=1, \ldots, N .
\end{gathered}
$$

Taking $\alpha_{k, l}$ out from bracket, it is put in the form of

$$
\begin{gathered}
\frac{\partial J\left(u^{h}\right)}{\partial \boldsymbol{\alpha}_{k, l}}=\left[\int_{D} p(x, y)\left(\sum_{k, l=1}^{N} \frac{\partial \boldsymbol{\phi}_{k, l}(x)}{\partial x}\right) \frac{\partial \boldsymbol{\phi}_{k, l}(x)}{\partial x} d D+\int_{D} q(x, y)\left(\sum_{k, l=1}^{N} \frac{\partial \boldsymbol{\phi}_{k, l}(x)}{\partial y}\right) \frac{\partial \boldsymbol{\phi}_{k, l}(x)}{\partial y} d D\right] \boldsymbol{\alpha}_{k, l}- \\
-\int_{D} f(x, y) \boldsymbol{\phi}_{k, l}(x) d D=0, \quad k, l=\overline{1, N} .
\end{gathered}
$$

Thus, according to (9) and (10) introducing the notation

$$
\begin{aligned}
a_{k, l}^{i, j} & =a_{N(k-1)+l, N(i-1)+j}=\int_{D} p(x, y)\left(\sum_{k, l=1}^{N} \frac{\partial \phi_{k, l}(x)}{\partial x}\right) \frac{\partial \phi_{i, j}(x)}{\partial x} d D+ \\
& +\int_{D} q(x, y)\left(\sum_{k, l=1}^{N} \frac{\partial \phi_{k, l}(x)}{\partial y}\right) \frac{\partial \phi_{i, j}(x)}{\partial y} d D, k, l, i, j=\overline{1, N},
\end{aligned}
$$

we get a system of linear algebraic equations (8).

$$
a_{k, l}^{k, l}, a_{k, l}^{k, l-1}, a_{k, l}^{k-1, l}, a_{k, l}^{k-1, l+1}, k, l=\overline{1, N} .
$$

Given the symmetry and block tridiagonal of matrix $A$, it is enough to define

I. Accroding (13), where $i, j=k, l$ we find $a_{k, l}^{k, l}$

$$
\begin{gathered}
a_{k, l}^{k, l}=\int_{D_{k, l}^{h}}\left[p(x, y)\left(\frac{\partial \boldsymbol{\phi}_{k, l}}{\partial x}\right)^{2}+q(x, y)\left(\frac{\partial \boldsymbol{\phi}_{k, l}}{\partial y}\right)^{2}\right] d D= \\
=\int_{D_{1}}\left[p(x, y)\left(\frac{\partial \boldsymbol{\phi}_{k, l}}{\partial x}\right)^{2}+q(x, y)\left(\frac{\partial \boldsymbol{\phi}_{k, l}}{\partial y}\right)^{2}\right] d x d y+\int_{D_{2}}\left[p(x, y)\left(\frac{\partial \boldsymbol{\phi}_{k, l}}{\partial x}\right)^{2}+q(x, y)\left(\frac{\partial \boldsymbol{\phi}_{k, l}}{\partial y}\right)^{2}\right] d x d y+ \\
+\int_{D_{3}}\left[p(x, y)\left(\frac{\partial \boldsymbol{\phi}_{k, l}}{\partial x}\right)^{2}+q(x, y)\left(\frac{\partial \boldsymbol{\phi}_{k, l}}{\partial y}\right)^{2}\right] d x d y+\int_{D_{4}}\left[p(x, y)\left(\frac{\partial \boldsymbol{\phi}_{k, l}}{\partial x}\right)^{2}+q(x, y)\left(\frac{\partial \boldsymbol{\phi}_{k, l}}{\partial y}\right)^{2}\right] d x d y+ \\
+\int_{D_{5}}\left[p(x, y)\left(\frac{\partial \boldsymbol{\phi}_{k, l}}{\partial x}\right)^{2}+q(x, y)\left(\frac{\partial \boldsymbol{\phi}_{k, l}}{\partial y}\right)^{2}\right] d x d y+\int_{D_{6}}\left[p(x, y)\left(\frac{\partial \boldsymbol{\phi}_{k, l}}{\partial x}\right)^{2}+q(x, y)\left(\frac{\partial \boldsymbol{\phi}_{k, l}}{\partial y}\right)^{2}\right] d x d y .
\end{gathered}
$$


We calculate the integrals in each of the areas $D_{k, l}^{h}$, taking into consideration form of basis function $\varphi_{k, l}(x, y)$ in considered area.

Further, all found six values are substituted in (15). In this case, we combine integrals with the same values and use the notation

$$
\begin{gathered}
\widetilde{P}_{k \pm \frac{1}{2}, l}=\frac{1}{h^{2}} \int_{D_{k, l}^{h} \cap D_{k \pm 1, l}^{h}} p(x, y) d x d y \\
\widetilde{Q}_{k, l \pm \frac{1}{2}}=\frac{1}{h^{2}} \int_{D_{k, l}^{h} \cap D_{k, l \pm 1}^{h}} q(x, y) d x d y
\end{gathered}
$$

It comes,

$$
\begin{aligned}
a_{k, l}^{k, l-1} & =\int_{D_{1} \cup D_{6}}\left(p(x, y) \frac{\partial \phi_{k, l}}{\partial x} \frac{\partial \phi_{k, l-1}}{\partial x}+q(x, y) \frac{\partial \phi_{k, l}}{\partial y} \frac{\partial \phi_{k, l-1}}{\partial y}\right) d x d y= \\
= & \int_{D_{1}}\left(p(x, y) \frac{\partial \phi_{k, l}}{\partial x} \frac{\partial \phi_{k, l-1}}{\partial x}+q(x, y) \frac{\partial \phi_{k, l}}{\partial y} \frac{\partial \phi_{k, l-1}}{\partial y}\right) d x d y+ \\
& +\int_{D_{6}}\left(p(x, y) \frac{\partial \phi_{k, l}}{\partial x} \frac{\partial \phi_{k, l-1}}{\partial x}+q(x, y) \frac{\partial \phi_{k, l}}{\partial y} \frac{\partial \phi_{k, l-1}}{\partial y}\right) d x d y
\end{aligned}
$$

The found values of the integrals, substituting in (19) and using the notation (17) we get

$$
\begin{aligned}
& a_{k, l}^{k, l-1}=-\frac{1}{h^{2}} \int_{D_{1}} q(x, y) d x d y-\frac{1}{h^{2}} \int_{D_{6}} q(x, y) d x d y= \\
& =-\frac{1}{h^{2}} \cdot \int_{D_{1} \cup D_{6}} q(x, y) d x d y=-\tilde{Q}_{k, l-\frac{1}{2}} \\
& a_{k, l}^{k-1, l}=\int_{D_{1} \cup D_{2}}\left(p(x, y) \frac{\partial \phi_{k, l}}{\partial x} \frac{\partial \phi_{k-1, l}}{\partial x}+q(x, y) \frac{\partial \phi_{k, l}}{\partial y} \frac{\partial \phi_{k-1, l}}{\partial y}\right) d x d y= \\
& =\int_{D_{1}}\left(p(x, y) \frac{\partial \phi_{k, l}}{\partial x} \frac{\partial \phi_{k-1, l}}{\partial x}+q(x, y) \frac{\partial \phi_{k, l}}{\partial y} \frac{\partial \phi_{k-1, l}}{\partial y}\right) d x d y+ \\
& +\int_{D_{2}}\left(p(x, y) \frac{\partial \phi_{k, l}}{\partial x} \frac{\partial \phi_{k-1, l}}{\partial x}+q(x, y) \frac{\partial \phi_{k, l}}{\partial y} \frac{\partial \phi_{k-1, l}}{\partial y}\right) d x d y .
\end{aligned}
$$

III. To find $a_{k, l}^{k-1, l}$ by formula (14), where $i, j=k-1, l$ we move vertex $p_{k, l}$ for one pitch to left and from fig.1. it is seen that $p_{k, l}$ and $p_{k-1, l}$ are the vertexes of triangles $D_{1}$ and $D_{2}$. It means, $a_{k, l}^{k-1, l}$ is defined in the triangles of $D_{1}$ and $D_{2}$.
Found values $a_{k, l}^{k-1, l}$, in the triangles $D_{1}$ and $D_{2}$ substitute in (21) with (16) and get

$$
\begin{aligned}
a_{k, l}^{k-1, l}= & -\frac{1}{h^{2}} \cdot \int_{D_{1}} p(x, y) d x d y-\frac{1}{h^{2}} \cdot \int_{D_{2}} p(x, y) d x d y= \\
& =-\frac{1}{h^{2}} \cdot \int_{D_{1} \cup D_{2}} p(x, y) d x d y=-\tilde{P}_{k-\frac{1}{2}, l}
\end{aligned}
$$

IV. It remains only to identify the element, $a_{k, l}^{k-1, l+1}$. For that the vertex $p_{k, l}$, needs to be moved firstly for one pitch to left and then for one pitch up. According to fig.1. it is seen that $p_{k, l}$ and $p_{k-1, l+1}$ are the vertexes of the triangles $D_{2}$ and $D_{3}$. But 
with the selected shift of $p_{k, l}$ the vertex $p_{k-1, l+1}$ would not belong to any of six triangles. Therefore

$$
a_{k, l}^{k-1, l+1}=0
$$

All found values (18), (20), (22), (23) substituting in (8), taking into account the symmetry of the matrix A we get

$$
\begin{gathered}
\left(\tilde{P}_{k-\frac{1}{2}, l}+\tilde{Q}_{k, l-\frac{1}{2}}+\tilde{P}_{k+\frac{1}{2}, l}+\tilde{Q}_{k, l+\frac{1}{2}}\right) \boldsymbol{\alpha}_{k, l}-\tilde{Q}_{k, l-\frac{1}{2}} \boldsymbol{\alpha}_{k, l-1}- \\
-\tilde{Q}_{k, l+\frac{1}{2}} \boldsymbol{\alpha}_{k, l+1}-\tilde{P}_{k-\frac{1}{2}, l} \boldsymbol{\alpha}_{k-1, l}-\tilde{P}_{k+\frac{1}{2}, l} \boldsymbol{\alpha}_{k+1, l} ; k, l=\overline{1, N}
\end{gathered}
$$

Allowing in (24)

$$
\begin{aligned}
\left(A_{1} \boldsymbol{\alpha}\right)_{k, l}= & -\tilde{P}_{k-\frac{1}{2}, l} \boldsymbol{\alpha}_{k-1, l}+\left(\tilde{P}_{k-\frac{1}{2}, l}+\tilde{P}_{k+\frac{1}{2}, l}\right) \boldsymbol{\alpha}_{k, l}- \\
& -\tilde{P}_{k+\frac{1}{2}, l} \boldsymbol{\alpha}_{k+1, l} ; k, l=\overline{1, N} \\
\left(A_{2} \boldsymbol{\alpha}\right)_{k, l}= & -\tilde{Q}_{k, l-\frac{1}{2}} \boldsymbol{\alpha}_{k, l-1}+\left(\tilde{Q}_{k, l-\frac{1}{2}}+\tilde{Q}_{k, l+\frac{1}{2}}\right) \boldsymbol{\alpha}_{k, l}- \\
& -\tilde{Q}_{k, l+\frac{1}{2}} \boldsymbol{\alpha}_{k, l+1} ; k, l=\overline{1, N}
\end{aligned}
$$

Then, using $A_{1}+A_{2}=A$, we get system (8)

\section{Variational methods for constructing iterative algorithms}

For numerical solution of system (6), one can apply variational type methods, such as, the method of rapid descent, the method of minimal corrections, the method of conjugate gradients, etc.

The conjugate gradient method is most preferable for systems with a self-adjoint positive matrix $A=A^{*}>0$. With poor conditioning of the matrix, this method does not always become computationally stable.

Operator $A$ is self-adjoint and positive definite operator in the space of grid functions $H_{h}^{0}$. Into $H_{h}^{0}$ we insert scalar product

$$
(u, v)=\sum_{i, j=1}^{N-1} u_{i, j} v_{i, j} h_{1} h_{2}
$$

and norm

$$
\|u\|=\sqrt{(u, u)} .
$$

To solve an equation of the form $A \alpha=g$ we use the conjugate gradient method. The iteration process is implemented in the following order:

I. Preparation before the iterative process

For given $\alpha_{i, j}^{0}$ the residual is calculated $s_{i, j}^{0}=r_{i, j}^{0}=\left(A \alpha^{0}\right)_{i, j}-g_{i, j} i=1, \ldots, n_{1}, j=1, \ldots, n_{2}$;

II. $k$-integration of the method

1) Calculate the parameter: $\gamma_{k}=\frac{\left(r_{i, j}^{k-1}, r_{i, j}^{k-1}\right)}{\left(A s_{i, j}^{k}, s_{i, j}^{k-1}\right)}$,

2) $r_{i, j}^{k}=r_{i, j}^{k-1}-\gamma_{k} A s_{i, j}^{k}$

3) The following approximation of the solution is calculated by the formula: $\alpha_{i, j}^{k}=\alpha_{i, j}^{k-1}-\gamma_{k} s_{i, j}^{k}$

4) Calculate the parameter: $\beta_{k}=\frac{\left(r_{i, j}^{k}, r_{i, j}^{k}\right)}{\left(r_{i, j}^{k-1}, r_{i, j}^{k-1}\right)}$

5) The auxiliary value is calculated by the formula: $s_{i, j}^{k+1}=r_{i, j}^{k}+\beta_{n} s_{i, j}^{k}$

where

$$
\begin{gathered}
\left(A s^{k}\right)_{i, j}=\frac{1}{h_{1}^{2}}\left[\tilde{P}_{i+\frac{1}{2}, j}\left(s_{i+1, j}-s_{i, j}\right)-\tilde{P}_{i-\frac{1}{2}, j}\left(s_{i, j}-s_{i-1, j}\right)\right]+ \\
+\frac{1}{h_{2}^{2}}\left[\tilde{Q}_{i+\frac{1}{2}, j}\left(s_{i, j+1}-s_{i, j}\right)-\tilde{Q}_{i, j-\frac{1}{2}}\left(s_{i, j}-s_{i, j-1}\right)\right] \\
i, j=\overline{1, N}
\end{gathered}
$$

$\backslash$ This process continues until the criterion for stopping the iterations $\left\|\alpha^{k+1}-\alpha^{k}\right\| \leq \varepsilon$ is satisfied.

\section{Calculation examples}

To illustrate the proposed method, we consider an example of the problem (11) - (12) in a circle.

Let in a rectangular area $D$ is a circle $\Omega$ with $\mathrm{R}$ radius and center $\left(c_{1}, c_{2}\right)$. It is required to find an approximate solution of the Dirichlet problem for the Poisson equation.

$$
\frac{\partial^{2} u}{\partial x_{1}^{2}}+\frac{\partial^{2} u}{\partial x_{2}^{2}}=-x_{1} \cdot x_{2}
$$


In the circle, under the condition

$$
\left.u\left(x_{1}, x_{2}\right)\right|_{\partial \Omega}=0
$$

Then

$$
\begin{gathered}
\bar{D}=\left\{\left(x_{1}, x_{2}\right), a_{1} \leq x_{1} \leq a_{2}, b_{1} \leq x_{2} \leq b_{2}\right\}, \\
\Omega=\left\{\left(x_{1}, x_{2}\right),\left(x_{1}-c_{1}\right)^{2}+\left(x_{2}-c_{2}\right)^{2} \leq R^{2}\right\}, \\
\partial \Omega=\left\{\left(x_{1}, x_{2}\right),\left(x_{1}-c_{1}\right)^{2}+\left(x_{2}-c_{2}\right)^{2}=R^{2}\right\} .
\end{gathered}
$$

Auxiliary problem of the fictitious domains method

$$
\begin{gathered}
\left\{\begin{array}{c}
\frac{\partial}{\partial x_{1}}\left(p\left(x_{1}, x_{2}\right) \frac{\partial u^{\varepsilon}}{\partial x_{1}}\right)+\frac{\partial}{\partial x_{2}}\left(q\left(x_{1}, x_{2}\right) \frac{\partial u^{\varepsilon}}{\partial x_{2}}\right)=-x_{1} \cdot x_{2}, \\
\left.u^{\varepsilon}\left(x_{1}, x_{2}\right)\right|_{\partial D}=0
\end{array}\right. \\
p\left(x_{1}, x_{2}\right)=q\left(x_{1}, x_{2}\right)=\left\{\begin{array}{l}
1,\left(x_{1}, x_{2}\right) \in \Omega, \\
1 / \varepsilon,\left(x_{1}, x_{2}\right) \in D / \Omega .
\end{array}\right. \\
f^{\varepsilon}\left(x_{1}, x_{2}\right)=\left\{\begin{array}{l}
-x_{1} \cdot x_{2},\left(x_{1}, x_{2}\right) \in \Omega \\
0,\left(x_{1}, x_{2}\right) \in D / \Omega .
\end{array}\right.
\end{gathered}
$$

Were conducted numerical experiments. Below is the table 1 for the number of iterations $N$ and calculation errors $\left\|u_{h}-u\right\|_{C}$ at $n_{1}=n_{2}=101$ and various meanings $\varepsilon$ for Ritz method.

Table 1 - Results of numerical experiments

\begin{tabular}{|c|c|c|c|c|}
\hline$n_{1}$ & $n_{2}$ & $1 / \varepsilon$ & $N$ & $\left\|u_{h}-u\right\|_{C}$ \\
\hline 101 & 101 & $10^{2}$ & 1221 & 0.003888 \\
\hline 101 & 101 & $10^{3}$ & 3395 & 0.003820 \\
\hline 101 & 101 & $10^{4}$ & 7920 & 0.003819 \\
\hline 101 & 101 & $10^{5}$ & 12737 & 0.003818 \\
\hline 101 & 101 & $10^{6}$ & 20042 & 0.003817 \\
\hline 101 & 101 & $10^{7}$ & 26592 & 0.003816 \\
\hline
\end{tabular}

The results show that with an increase in the number of grid nodes, the error in the solution decreases. In fig. 2 shown the result of solving the problem using an explicit difference scheme with $\varepsilon_{*}=\varepsilon=10^{-4}$ on a uniform grid with a size of 101 $\times$ 101. Therefore, the original system of linear algebraic equations has $101 \times 101$ unknowns.

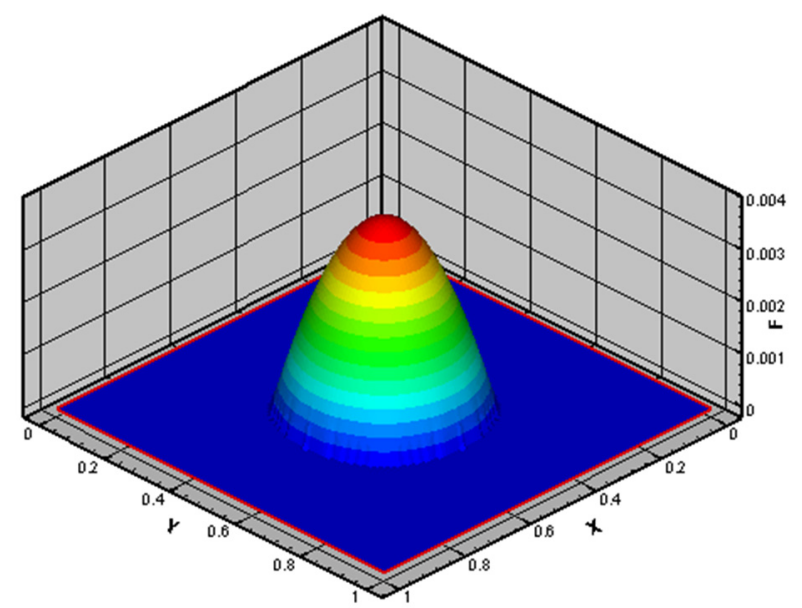

Figure 2 - Solution of the Dirichlet problem for the Poisson equation in a circle

\section{Conclusion}

Today, the task of developing and modifying numerical methods remains relevant. However, the development process of computing technology shifts the emphasis from the creation of new numerical methods to the study and classification of old ones in order to identify the best. Now for modern powerful computers, such characteristics as the amount of required memory, and the number of arithmetic operations are not necessarily in the foreground. More preferred are those methods that are distinguished by the ease of implementation on a computer, and allow to solve a wider class of problems.

The special advantages of this method are its simplicity and low memory costs, which makes it effective in solving large-scale problems.

The results of the computational experiment confirm the efficiency of the proposed method for solving the Dirichlet problem for the Poisson equation and its rather high efficiency.

\section{References}

1. Marchýk G.I. "Metody vychislitelno1 matematık1." Naýka (1989): 608.

2. Mýhambetjanov A.T., Otelbaev M.O., Smagýlov Sh.S. "Ob odnom novom prıblijennom 
metode reshenie nelıneinyh kraevyh zadach." Vychislitelnye tehnologi1 3, no. 4 (1998).

3. Samarskii A.A. "Teorna raznostnyh shem." Naýka (1983): 616.

4. Bakhvalov N.S, Zhidkov N.P, Kobelkov G.M. Chislennye metody - Izd.: Binom (2008).

5. N.M. Temırbekov, J.R. Jaksylykova. "Ob iteratsionnom metode fiktıvnyh oblaste1 dlia reshenı1a ellıptıcheskogo ýravnenı1a v oblastıah so slojno1 geometrie1." Izvestı1a MKTÝ 1m. H.A.Iasavı, Serıı matematıka, fizıka, informatıka. 1, no. 1(4) (2018): 128-132.

6. N.M.Temirbekov, Zh.R.Zhaksylykova. "AnIterative Method for Solving Non linear NavierStokes Equationsin Complex Domains Taking into Account Boundary Conditions with Uniform Accuracy." AIP Conference Proceedings 1997, 020036 (2018); doi: 10.1063/1.5049030.

7. Temirbekov A.N., Wójcik W. "Numerical Implementation of the Fictitious Domain Method for Elliptic Equations." International Journal of Electronics and Telecommunications 60, no. 3 (2014): 219-223.

8. Temirbekov A.N. "Numerical implementtation of the method of fictitious domains for elliptic equations." 3rd International Conference on Analysis and Applied Mathematics, ICAAM 1759 (2016): 020053-1-020053-6; doi: 10.1063/1.4959667.

9. Kireev V.I., Panteleev A.V., Chislennye metody $\mathrm{V}$ primerakh $\mathrm{i}$ zadachakh. Uchebnoe posobie. 4-e izd., ispr.-SPb.: Izdatel'stvo «Lan'» (2015): 488.

10. Samarskii A. A., Nikolaev E. S. "Metody reshenia setochnyh uravnenii." Naýka (1978): 592.

11. Bykov V.M., Nizameev Kh.R. Variatsionnye metody $\mathrm{v}$ mekhanike sploshnykh sred: Metodicheskie ukazaniya po spetskursu. Chelyabinsk: Chelyabinskiy gos. un-t (2001): 36.

12. Berezin I.S., Zhidkov N.P. "Metody vychisleniy." GIFML 2 (1959): 620. 